\title{
TITLE:
}

\section{Japanese Macaques Depend not Only on Neighbours but also on More Distant Members for Group Cohesion}

\section{$\operatorname{AUTHOR}(\mathrm{S}):$}

Sugiura, Hideki; Shimooka, Yukiko; Tsuji, Yamato

\section{CITATION:}

Sugiura, Hideki ...[et al]. Japanese Macaques Depend not Only on Neighbours but also on More Distant Members for Group Cohesion. Ethology 2013, 120(1): 21-31

\section{ISSUE DATE:}

2013-10-27

URL:

http://hdl.handle.net/2433/196659

\section{RIGHT:}

(c) 2013 Blackwell Verlag GmbH.; This is the peer reviewed version of the following article: Sugiura, H., Shimooka, Y., Tsuji, Y. (2014), Japanese Macaques Depend not Only on Neighbours but also on More Distant Members for Group Cohesion. Ethology, 120:21-31., which has been published in final form at http://dx.doi.org/10.1111/eth.12176; この論 文は出版社版でありません。引用の際には出版社版をご確認ご利用ください。; This is not the published version.

Please cite only the published version. 
Authors:

Hideki Sugiura (Corresponding author) Wildlife Research Center, Kyoto University

2-24 Tanaka-Sekiden-cho, Sakyo, Kyoto, 606-8203, Japan

E-mail: sugiura.hideki.7s@kyoto-u.ac.jp

Telephone: +81-75-771-4379

Fax: +81-75-771-4394

Yukiko Shimooka

Department of Environmental Science, Teikyo University of Science and Technology 2525 Yatsusawa, Uenohara, Yamanashi 409-0133, Japan

Title: Japanese macaques depend not only on neighbours but also on more distant members for group cohesion

$$
\text { Fax: }+81-75-771-4394
$$

2

Primate Research Institute, Kyoto University

Kanrin 41, Inuyama, Aichi 484-8506, Japan

Running title: Behavioral mechanism for group cohesion in Japanese macaques (1)

Word count: 7064 words 


\section{Abstract}

A well-known behavioural model for group aggregation is that an individual depends on a few neighbouring individuals to adjust its movement, such as departure (repulsion) from and approach (attraction) to neighbours. However, an individual may rely not only on a few closest neighbours, but also on more distant individuals, in a group of stable membership. We measured temporal changes in the local density of individuals around a focal individual and changes in distance to other focal individuals in a group of wild Japanese macaques to determine whether the macaques depended only on a few neighbours or also on more distant individuals for adjustments in cohesiveness. We used simultaneous focal animal sampling, with two observers recording the individuals’ locations using a global positioning system (GPS), over three seasons. Numbers of individuals within $20 \mathrm{~m}$ from an animal tended to increase after 10 min when there were a small number of individuals around the animal. However, the number tended to decrease when there was a larger number of individuals. It remained similar when there were an intermediate number of individuals. The two focal animals tended to separate after 10 min when the interindividual distance was short. However, they tended to move closer when far apart. They remained a similar distance apart when they were at an intermediate distance. Contact calls, which are suggested to function as locating group members and keeping cohesiveness, were emitted more frequently when the distance between the two focal animals was very large in two seasons. However, the rate of contact calls was not influenced by the number of individuals within $20 \mathrm{~m}$ from an animal. These results suggest that individual Japanese macaques do not only rely on a few closest neighbours, but also on more distant group members. Japanese macaques may know the general whereabouts of the whole group, and when they stay at the periphery of the group, they may emit contact calls frequently and move towards the central zone so as not to become separated from the group. 


\section{Introduction}

Many social animals exhibit cohesive aggregation, such as insect swarming, fish schooling, bird flocking, and mammalian herding (Cavagna et al. 2010; Schellinck \& White 2011). While maintaining a close distance to others is favourable for predation avoidance (Hamilton 1971), it is unfavourable for foraging because of competition among group members (e.g., Wrangham, 1980). The cost of grouping may be mediated by adjusting an individual's proximity to other group members (Aureli et al. 2008), raising the question of how animals adjust proximity and cohere as a group.

Theoretical studies have proposed models of individual movement strategies explaining collective group motion in which an animal monitors the locations and movements of a few closest neighbours and adjusts its movements to those of those neighbours. For example, when an individual is too close to its neighbour, it travels in the opposite direction (repulsion) and when an individual is far from its neighbour, it moves towards the neighbour (attraction) (Schellinck \& White 2011).

Such models provide simple mechanisms of collective movement, which require an animal to know the locations of other animals within a close distance, but not those of all members of the group. However, some other behavioural processes may be at work during collective movement in social animals that form small groups with stable memberships and have high cognitive ability, such as primates (Aureli et al. 2008; Shimooka 2003) and dolphins (Karczmarski et al. 2005). One possible behavioural process is that an individual relies not only on a few close neighbours, but also on individuals more distant in location and/or movement of the whole group. To examine this, we observed adjustments of proximity among Japanese macaques (Macaca fuscata) in the wild.

Japanese macaques form female philopatric groups. Females reside in the natal group, 
whereas males emigrate out of the group at adolescence (Yamagiwa \& Hill 1998). There is a linear dominance hierarchy, based on kin relationships, between resident females (Hill \& Okayasu 1995), and the groups are relatively cohesive with stable membership. The group’s habitat and activities vary seasonally (Tsuji et al. 2006). It has been suggested that the frequently emitted contact call, or “coo call” (Green 1975), is an important means of maintaining group cohesiveness (Koda \& Sugiura 2010; Suzuki \& Sugiura 2011). Japanese macaques are known to change their behaviour, such as monitoring other individuals, depending on the proximity to nearby members (Suzuki \& Sugiura 2011). Thus, it is likely that they rely on the neighbours for the adjustment of proximity, however, it is possible that Japanese macaques also may rely on more distant animals. Although the data on proximity to distant animals is quite limited, they may locate approximate position of the whole group (Wada \& Matsuzawa 1986).

As the first aim of this study, we examined a basic assumption that Japanese macaques adjust proximity to group members. If they do, proximity to group members is expected to increase when they are far apart and vice versa. In particular, we predicted that 1 ) the number of individuals within $20 \mathrm{~m}$ of an animal would increase and/or 2) distance to the other focal female would decrease (both indicating increased proximity to group members), if an animal is far apart from group members and vice versa.

Our second aim is to examine whether Japanese macaques rely on the neighbour individuals and/or more distant individuals for their adjustment of proximity. If they adjust proximity relying only on neighbours, change (i.e., increase and decrease) in proximity to group members is expected to be accounted for by initial state of proximity to the neighbours. In this case, we predicted that 1) variance in change in number of individuals within $20 \mathrm{~m}$ and 2) variance in change in distance to the other focal individual would largely be accounted for by 
the initial number of individuals within $20 \mathrm{~m}$. Alternatively, they may rely not only on neighbours but also on more distant individuals. In this case, we predicted that variances in these two response variables would be accounted for both by the initial number of individuals within $20 \mathrm{~m}$ and also by the initial distance to the other focal animal.

We also examined the rate of contact calls with the same analytical design to explore the possibility that macaques change behaviour related to group aggregation depending not only on close neighbours but also on more distant animals.

We propose that macaques rely on both close neighbours and more distant animals and that they know the location of the whole group and adjust their movements based on such information. In addition, we discuss possible mechanisms and functions of adjustment of proximity.

\section{Methods}

Subjects

We studied the A group of wild Japanese macaques on Kinkazan Island, northern Japan $\left(38.30^{\circ} \mathrm{N}, 141.56^{\circ} \mathrm{E}\right.$; elevation $\left.0-445 \mathrm{~m} \mathrm{ASL}\right)$. Members of the A group have been identified individually since 1983 and are used to human observers (Sato 1988). No natural predator of these monkeys exists on the island and hunting is forbidden by law and religion. During the study period, the subject group included 31-39 individuals: 17 adult females ( $\geq 5$ years old), 5-13 adult males ( $\geq 5$ years old), $2-5$ juvenile females (1-4 years old), $2-4$ juvenile males (1-4 years old), and 5-8 infants ( $<1$ year old). The macaques' range covered approximately $3 \mathrm{~km}^{2}$ during the study period. Details of the subjects and their habitat have been described elsewhere (Tsuji et al. 2006; Tsuji \& Takatsuki 2004; Tsuji \& Takatsuki 2012).

We collected data during three seasons: autumn (16 October to 8 November, 2003), 
winter (10-22 February, 2004), and summer (6-23 July, 2004). Observation was conducted in 6:30-16:40 in autumn, 7:15-16:55 in winter, and 6:15-18:30 in summer. Data were collected almost equally during these hours. We chose 10, 9, and 7 females of varying dominance ranks as the subjects in the autumn, winter, and summer, respectively. Seven of the animals were observed during all three study periods. The mean observation times per individual were 33.1 $\pm 5.0,31.9 \pm 3.5$, and $29.1 \pm 1.3 \mathrm{~h}$ in the autumn, winter, and summer, respectively. We observed 30 of 45 possible pairs in autumn, 29 of 36 possible pairs in winter, and all 21 possible pairs in summer. The mean observation times per pair were $7.3 \pm 0.1$ (mean $\pm S E$ ), $9.3 \pm 0.1$, and 4.4 $\pm 0.1 \mathrm{~h}$ in the autumn, winter, and summer, respectively.

\section{Data Collection}

Two observers followed each anoestrous focal adult female using the focal animal sampling method. We excluded oestrous females from the study because they tended to stay on the periphery of the group to mate with low-ranking or non-troop males (Hayakawa 2007).

Each observation session lasted $4 \mathrm{~h}$, during which we recorded the number of coo calls (Green 1975; Sugiura 2007b) emitted by each focal animal per minute. We recorded the number of individuals within $20 \mathrm{~m}$ of each focal animal in 5-min intervals by instantaneous sampling. We excluded infants $<1$ year of age because they were usually dependent on other individuals. We excluded data when visibility was $<20 \mathrm{~m}$.

We measured location, time, and the positional dilution of precision (PDOP, a measurement of position accuracy) using a GPS receiver (IPS-5100; Sony, Tokyo, Japan or GPS Pathfinder Pocket; Trimble, Sunnyvale, CA, USA). We recorded GPS data every $1 \mathrm{~s}$ on a handheld computer (200LX or iPAQ h2210; Hewlett-Packard, Tokyo, Japan). We typically remained within a horizontal distance of $10 \mathrm{~m}$ from the focal animal and considered the position 
of the observer to be that of the focal animal. We excluded GPS data recorded when the focal animal was out of view or was $>15 \mathrm{~m}$ away for $>1 \mathrm{~min}$.

Analysis

We used two measures of proximity, covering different ranges of distance. One is proximity to group members at close range $(0-20 \mathrm{~m})$. We measured the number of individuals within $20 \mathrm{~m}$ from the focal adult female (i.e., the density of individuals around an animal) and its change after $10 \mathrm{~min}$. Another is proximity to a group member over a longer distance ( $\geq 40 \mathrm{~m})$. We measured the distance to the other focal individual observed simultaneously with GPS (i.e., the distance between two random adult females in a group) and its change after 10 min.

We converted location data into rectangular coordinates using universal traverse mercator (UTM) projection. To remove large location errors, we used only measurements with a PDOP smaller (better) than 6.5 with a three-dimensional fix (D'Eon \& Delparte 2005). We chose the location data with the smallest PDOP within a window of -15 to $+15 \mathrm{~s}$ from the sampling time. On occasion, three observers simultaneously followed a focal animal. In these cases, we used the distance between each pair combination (e.g., A-B, A-C, B-C) as independent data.

We examined three response variables as behavioural correlates of adjustment of proximity: 1) change in number of individuals, 2) change in distance between two focal animals, and 3) contact call (coo call) rate. We tested whether the variance in each of these three response variables was influenced by two explanatory variables: 1) initial number of individuals within $20 \mathrm{~m}$ from a focal animal and 2) initial distance to the other focal animal.

Changes in numbers of individuals and in distance to the other focal animal were sampled in 15-min intervals (at a minimum). Call rate was sampled in 5-min intervals (at a minimum). 
The sampling interval was extended by 5 min (i.e., 20, 25, $30 \mathrm{~min}$ ) if loss of the focal animal, loss of the number of individuals around the focal animal, or unreliable GPS positions occurred.

We performed all analyses separately in each of the three seasons, because the cohesiveness of the group varied largely with season (Sugiura et al. 2011). Our main interest was to examine whether the adjustment of proximity occurs in each season, as opposed to being due to merely seasonal variation in proximities among group members. Thus, we conducted analysis in each season to simplify the design of statistical analyses. Interindividual distances were extremely large (0-1225 m) and subgrouping occurred during the summer (Sugiura et al. 2011). We divided the distances into three categories of non-subgrouping, subgrouping, and unknown, following a previous study (Sugiura et al. 2011). We used data categorised as non-subgrouping and subgrouping separately in the present analysis. Our main objective was to examine the adjustment of proximity in normal grouping (i.e., non-subgrouping). However, behavioural differences between grouping and subgrouping were also of interest, because the differences may reveal characteristics of grouping. Thus, we also analysed the behaviour during subgrouping.

We used data when the initial distance to the other focal individual was $\geq 40 \mathrm{~m}$. Two explanatory variables, initial number of individuals within $20 \mathrm{~m}$ of the focal animal and initial distance, correlated weakly with each other, when we included all the data. However, this correlation disappeared, excluding the data where the distance to the other focal animal was $<40 \mathrm{~m}$. Our main objective was to examine which of the explanatory variables accounted for response variables; thus, we excluded data where the effects of the two factors were difficult to separate. This procedure tends to exclude instances where the group spread was small. The change in number of individuals was calculated as (number of individuals after 10 min) - (initial number of individual). Thus, a positive value indicates that the local density of 
individuals around the focal animal increased (increase in proximity), whereas a negative value indicates a decrease in the local density of individuals (decrease in proximity). The change in distance was calculated as (initial distance) - (distance after $10 \mathrm{~min}$ ). Thus, a positive value indicates approach (increase in proximity), whereas a negative value indicates greater separation (decrease in proximity). Call rate was the number of coo calls made by the focal animal from 0 to $+1 \mathrm{~min}$, where we measured the number of individuals around the focal animal at $0 \mathrm{~min}$.

To analyse changes in numbers of individuals and in distance, two explanatory variables (initial numbers near the focal animal and distance to the other focal animal) were treated as continuous variables. To analyse contact call rate, we treated one explanatory variable (distance to the other focal animal) as a discrete variable to describe the general trend in coo call rate at different interindividual distances, because our exploratory analyses revealed that the rate of coo calls did not change linearly with the initial distance, but increased especially at long distances.

Statistical analyses were conducted using generalised linear mixed models and the SAS software (ver. 9.2; SAS Institute, Cary, NC). We used a normal distribution to analyse changes in numbers of individuals and changes in distances, and a generalised Poisson distribution to analyse coo call rates. Analyses of changes in numbers of individuals and rates of coo calls were performed on an individual basis. For the analysis, we treated the focal individuals as a random factor. Analysis of changes in distance was performed on a pair basis, because this response variable was derived from the positions of the two focal animals. For this, we used two explanatory variables; 1 ) initial number of individuals around the focal animals as an average of the numbers of individuals around the two focal animals, and 2) initial distance between the two focal animals. We treated the focal pair was a random factor. Post hoc pairwise comparisons 
were conducted for the analyses of coo call rate, using the Bonferroni correction.

\section{Results}

Changes in numbers of individuals at a close distance

We examined the changes in the number of individuals within $20 \mathrm{~m}$ from the focal animal after 10 min (Fig. 1). A positive value indicated that the number of individuals increased (increase in proximity), a negative value indicated that it decreased (decrease in proximity), and a value of zero indicated that it remained the same. Changes in number of individuals were influenced negatively by the initial number of individuals in each of the three seasons during non-subgrouping; that is, when the number of individuals around the focal animal was small, the number of individuals tended to increase after $10 \min$ (Fig. 1, autumn, $F_{1,9}=21.9, p=0.001$; winter, $F_{1,8}=23.0, p=0.001$; summer $\left.F_{1,6}=43.1, p=0.0006\right)$. The same tendency was observed during subgrouping in the summer, where each of the two focal individuals was in a separate subgroup $\left(F_{1,6}=56.7, p=0.0003\right)$.

By contrast, the changes in the numbers of individuals were not affected by the initial distance to the other focal animal in any season, including in subgrouping in the summer (autumn, $F_{1,9}=0.08, p=0.79$; winter, $F_{1,8}=0.76, p=0.41$; summer, non-subgrouping, $F_{1,6}=$ 4.97, $p=0.06$; summer, subgrouping, $F_{1,6}=1.48, p=0.27$ ).

\section{Changes in Distance}

We examined changes in distance after 10 min between two individuals (Fig. 2). A positive value indicated that the distance decreased (increase in proximity to the other focal individual), a negative value indicated that it increased (decrease in proximity), and a value of zero indicated that it remained the same. Changes in distance were influenced positively by the 
initial distance during non-subgrouping. When the distance to the other focal animal was larger, they tended to come closer after $10 \mathrm{~min}$ (Fig. $2 \mathrm{~A}-\mathrm{C}$, autumn, $F_{1,24}=28.2, p<0.0001$; winter, $F_{1,28}=52.6, p<0.0001$; summer, non-subgrouping, $\left.F_{1,18}=10.8, p=0.004\right)$. This tendency was not observed during subgrouping in the summer (Fig. 2D, $F_{1,13}=2.0, p=0.18$ ).

In contrast, variance in changes in distance were not accounted for by the initial number of individuals around the focal animal in any season, including in subgrouping in the summer (autumn, $F_{1,21}=1.99, p=0.17$; winter, $F_{1,28}=1.18, p=0.29$; summer, non-subgrouping, $F_{1,18}=$ 1.09, $p=0.31$; summer, subgrouping, $F_{1,11}=3.32, p=0.10$ ).

\section{Rate of Contact Calls}

We examined whether the contact call rates of focal animals varied based on the distance from another focal animal and on the number of individuals around the focal animal. In the autumn, the effect of distance apart was significant but that of the number of individuals was not (Fig. 3A, distance, $F_{4,41}=2.66, p=0.046$; number of individual, $F_{1,9}=0.18, p=0.68$ ). Pairwise comparisons revealed no significant difference between any pair. However, call rates tended to be higher with an interindividual distance of 90-230 m, versus those of 40-50 m ( $p=0.06)$.

Also in the winter, the effect of distance apart was significant but that of the number of individuals was not (Fig. 3B, distance, $F_{7,63}=3.60, p=0.003$; number of individual, $F_{1,8}=$ 0.0001, $p=0.99$ ). Pairwise comparisons revealed that call rates were significantly higher for interindividual distances of 120-270 m, compared to distances of 40-50 m and 60-70 m ( $p<$ $0.05)$.

In the summer, none of the effects was significant during non-subgrouping (distance, $F_{11,72}=0.74, p=0.70$; number of individual, $F_{1,6}=1.53, p=0.26$ ) or subgrouping (distance, $F_{8,45}=0.24, p=0.98$; number of individual, $\left.F_{1,6}=0.61, p=0.43\right)$. 
Discussion

Adjustment of Proximity to Close Neighbours

In our observations, the number of individuals within $20 \mathrm{~m}$ of the focal individual tended

to increase after a short period of time when there was a small number of individuals around her

and tended to decrease when there was a larger number of individuals in each of three seasons, including when the two focal animals were in different subgroups. In contrast, the change in number of individuals was not accounted for by the distance to the other focal individual.

These results suggest that Japanese macaques adjust their proximity to group members relying on the local density of group members within a close distance. Japanese macaques increased the number of neighbours, i.e., increased proximity, when they were far apart and vice versa. This adjustment appears to be a negative feedback of local density that may stabilize it. In this sense, this adjustment may be similar to attraction - repulsion movement toward the neighbours, and may be a common mechanism of aggregation with those of other animals, such as fish school (Aoki 1982) and bird flocks (Lukeman et al. 2010).

The mean number of individuals within $20 \mathrm{~m}$ of a focal animal (when the distance to the other focal animal was $\geq 40 \mathrm{~m}$ ) was 3.1 in autumn, 2.0 in winter, 1.4 in non-subgrouping in summer, and 1.3 in subgrouping in summer. Thus, the local density of individuals around focal animals was high in autumn, middle in winter and low in summer. Although the density of animals differed among seasons, adjustment of proximity to neighbours was constantly observed in each of the three seasons.

The mean visibility in the subject group’s habitat is ca. 30-50 m (Koda et al. 2008); thus, Japanse macaques are likely to keep sight of group members within a close distance, ca. $20 \mathrm{~m}$. It 
has been suggested that they monitor group members visually and adjust their movements to keep cohesiveness with other group members (Koda \& Sugiura 2010; Suzuki \& Sugiura 2011). Such adjustment continued even when the group was split into subgroups in the summer. This is consistent with the view that the group is split into two (or a few) subunits that move collectively with group members.

\section{Adjustment of Proximity to Distant Individuals}

The distance to the other focal animal also tended to decrease after a short period of time when the distance was longer and tended to increase when the distance was shorter in each of three seasons, except in subgrouping in the summer. This change of distance, however, was not accounted for by the numbers of individuals within $20 \mathrm{~m}$ from the focal animals.

These results suggest that Japanese macaques adjust their proximity to group members at relatively far distances. Focal pairs of macaques tended to separate after a short period of time when they were close together and tended to move closer when they were farther apart. This indicates that individual Japanese macaques move to cohere with group members at relatively far distances. This adjustment may also be a negative feedback of distance to far individuals, which may stabilize the proximity to distant group members.

The mean distance between the two focal individuals (when the distance was $\geq 40 \mathrm{~m}$ ) was $58.2 \mathrm{~m}$ in autumn, $72.0 \mathrm{~m}$ in winter, $85.1 \mathrm{~m}$ in non-subgrouping in summer, and $365.8 \mathrm{~m}$ in subgrouping in summer. Thus, the distance between two individuals was short in autumn, middle in winter and long in summer. Although the distance differed among seasons, adjustment of proximity to distant individuals was constantly observed in each of the three seasons, except in subgrouping in summer.

Such movement was not accounted by the local density of group members within a close 
distance. Because the distance to the other focal animal and the number of individuals within 20 m was not correlated, they might not be able to know the distance to the other focal animal from the local density of animals around them. These changes were observed even when the distance to the other focal animal was more than $40 \mathrm{~m}$. Considering visibility in the habitat, it seems unlikely that a focal individual directly monitored the position and movement of the other focal individual. This tendency, however, disappeared when the two animals were in separate subgroups. On such occasions, Japanese macaques lost the location of the other subgroup and their movements became independent of each other.

However, it is possible that Japanese macaques depend only on nearby individuals but not distant members. An alternative explanation is that Japanese macaques monitor more subtle information from the nearby individuals, which enable them to adjust proximity to distant individuals. A possible cue is temporal change of proximity of nearby group members, which may reflect group cohesiveness. However, change in local density of individuals in $10 \mathrm{~min}$ (average change in number of individuals within $20 \mathrm{~m}$ from the two focal animals from 0 to +10 min) did not explain the variance in change in distance between two focal individuals (Sugiura et. al., unpublished data). There are several possible cues from nearby individuals, e.g., timing and amount of change in proximity, activity of nearby individuals and social relationships to nearby individuals. In the present study, we could not record these detailed information of neighbour individuals in a natural setting. Continuous observation of nearby individuals, such as video recording would be applicable to further examinations.

In addition, we analyzed the change in distance on a pair basis, and used the numbers of nearby group members of the two focal individuals (average number) as an explanatory variable. However, an individual can know its own nearby members but not that of the other distant focal animal. Thus, the average number of nearby group members is a relatively rough 
approximation and it might have reduced the effect of nearby group members on the change of distance. This limitation can be improved if we can analyze this effect on an individual basis, e.g., discriminating one of the two animals that is more responsible for the change of distance.

\section{Contact Calls at Different Proximity}

Our results show that the coo call rate increased, especially for the longest distance class, in the autumn and winter. The coo call rate, however, was not influenced by the number of individuals within $20 \mathrm{~m}$ in any season. When the distance between two animals fell in the largest distance class, one or both focal animal(s) was likely to stay at the periphery of the spread group. Thus, the macaques seemed to emit contact calls frequently in the peripheral zone to contact group members. Therefore, Japanese macaques are apparently aware of which group zone they are in.

Such use of the contact call seems quite reasonable, considering the function of coo calls. The coo calls of Japanese macaques have been suggested to maintain group cohesiveness (Itani 1963; Mitani 1986) and are often emitted by individuals that may be likely to become separated from the group (Koda \& Sugiura 2010; Suzuki \& Sugiura 2011). Similar usage of contact calls are found in other species of primates. Chacma baboons (Papio cynocephalus) emit contact calls more frequently when they were separated from the group (Rendall et al. 2000). White-faced capuchin monkeys (Cebus capucinus) emit contact calls more often at the peripheral zone of the group and the calls are likely to relate to group movement (Boinski \& Campbell 1995).

Coo calls are, however, often elicited by the other's coo calls as vocal response (Sugiura 2007a). Thus, increase of coo call rate might have caused by the increase of coo calls of the other individuals. Because we did not record the vocalization from the other individuals, in this 
study, we can not examine the effect of other's call. Further observation should be needed, recording the rate of calls from other individuals and discriminating between spontaneous call (i.e., without preceding calls of the other individual) and response call (i.e., with preceding calls of the other individual).

We failed to detect difference in call rate in summer. The mean coo call rate per minute (when the distance to the other focal animal was $\geq 40 \mathrm{~m}$ ) was 0.36 in autumn, 0.22 in winter, 0.54 in non-subgrouping in summer, and 0.44 in subgrouping in summer. In summer, subject females produced coo calls frequently, probably because the group dispersed and they needed vocal contact most in this season. In this season, they may emit coo calls so frequently at any distance that they do not emit additional coo calls when they stayed far from the other focal animals.

\section{Possible Mechanisms of Adjustment of Proximity to Distant Individuals}

Japanese macaques may somehow locate the general whereabouts of the entire group. Assuming that macaques are aware of their proximity to their group’s central zone, individual movement from the peripheral zone towards the central zone may explain the more rapid approach from greater interindividual distances apart.

One possible mechanism for knowing the general location of the entire group is monitoring the movements of nearby group members. It appears difficult for an individual to see all of the group members directly, because it spreads over a wider area than one can see in the forest. In such conditions, movements of nearby group members may help to know the general location of the entire group. For example, if an individual remembers that most group members passed ahead of it, it ascertains that it is in a peripheral position in the group. Such a mechanism is plausible, considering their cognitive abilities (Roberts 2002) and that Japanese 
macaques usually travel straight to food sources (Maruhashi et al. 1998). Another possible mechanism is vocal communication. Audible range of contact calls is usually wider than visible range in forest, and contact calls from group members appears to help an individuals to locate the whole group. If they locate the general whereabouts of the entire group, they may change behaviour in relation to their own spatial position, e.g., central - peripheral zone of the group (Janson 1990; Robinson 1981). Further examination of behavioural changes in relation to relative position of the group is necessary, such as monitoring behaviour of group members (Kazahari \& Agetsuma 2010; Suzuki 2011) and contact calls (Boinski \& Campbell 1995; Rendall et al. 2000; this study).

Another possible mechanism for knowing the location of the group is that group members share knowledge of food resources and aggregate at a food patch (Ramos-Fernandez et al. 2006). If we assume that each individual accurately knows information of food resources and knows where group members go next, they can aggregate at such places without locating the whole group. However, it is unlikely that group members share the prior knowledge of food resources to such degree that they can forecast the other members' destinations. Aggregating at a food patch may help them to cohere, but monitoring the position of the group should be needed.

In addition, Japanese macaques do not always aggregate at a large food patch, especially in lean seasons. In the subject group, they feed on clumped food such as fruiting trees for about $80 \%$ of feeding time in autumn (Sugiura et al. 2011), where aggregating at such food patches may be possible. However, they feed on scattered food such as herb for about $85 \%$ and $63 \%$ of feeding time in winter and in summer, respectively (Sugiura et al. 2011), where they feed alone or with a few members. In such ecological condition, aggregating at a food patch would be difficult. In spite of these drastic changes of food availability, we observed a significant effect of 
initial distance of the focal pair on the change in distance between them, in different seasons. Thus, aggregating at a food patch alone can not account for group cohesion, although this should also help them to cohere.

In this study, the subjects did not engage in a particular activity, but did in various ones when we sampled their movements. The proportion of activities also varied in different seasons. In spite of this variation, the adjustment of distance between two distant animals was constantly observed in each of the three seasons. Thus, it is unlikely that adjustment of proximity is derived from a particular situation, such as aggregating at food patches. In autumn, the proportions of activities of subject animals were $17.0 \%$ in grooming, $12.4 \%$ in resting, $54.2 \%$ in foraging, $15.7 \%$ in moving and $0.7 \%$ in agonistic interaction. In winter, the proportions were $11.1 \%$ in grooming, $6.4 \%$ in resting, $72.7 \%$ in foraging, $9.4 \%$ in moving and $0.3 \%$ in agonistic interaction. In summer, the proportions were $11.1 \%$ in grooming, $21.5 \%$ in resting, $32.9 \%$ in foraging, $34.4 \%$ in moving and $0.3 \%$ in agonistic interaction.

\section{Possible Function of Adjustment of Proximity to Distant Individuals}

A major cost of grouping is feeding competition with group members (van Schaik \& van Noordwijk 1986), but adjusting proximity to group members can mediate the feeding competition. Adjusting proximity not only to members in close proximity but also to more distant members should bring more flexible group cohesion and foraging tactics. If we assume that Japanese macaques keep a particular distance to a few neighbours, they should need to synchronize their arrival and leave of a food patch with their neighbours. Such adjustment of proximity to neighbours should be a firm means for cohesion but are likely to affect feeding competition.

Actually, however, Japanese macaques seem to flexibly change their proximity and group 
cohesiveness. They change their proximity to group members depending on the quality of food (Sugiura et al. 2011). In addition, they do not always synchronize their foraging (Agetsuma 1995) or timing of leave from food patches (Kazahari \& Agetsuma 2010). Japanese macaques appear to adjust proximity to distant members, probably by locating approximate position of the whole group. This may bring them more freedom of positioning themselves and thus, freedom of food choice with lesser competition and/or better feeding efficiency. Predation free condition in this study should contribute to such flexible adjustment of proximity. Lack of predation may reduce the necessity of their keeping cohesiveness with nearby individuals, and made the adjustment proximity to distant individuals more detectable.

The present study suggests that Japanese macaques adjust their proximity to group members, relying not only on neighbouring group members, but also on more distant animals. Although adjustment of proximity to neighbours have been reported in a variety of species, adjustment of proximity to distant group members may be unique to group living animals that form a relatively small group with stable memberships, like Japanese macaques. However, it is still possible that Japanese macaques rely only on the information from nearby group members, and it accounts for the change in distance between two distant individuals. Further examination of the influence of nearby group members on the change of distance should be necessary, such as their activities, movements and coo calls. Examination from another view point would also possible. If they relay also on the distant group members, they should know the location of the whole group and adjust their movements based on such information. Examination of this possibility would also help to elucidate our hypothesis and to understand the mechanism of group cohesion in Japanese macaques and other group living animals. 
457

458

459

460

461

462

463

\section{Acknowledgements}

We thank Prof. K. Izawa for supporting our overall research activities on Kinkazan Island, the staff of Kinkazan Koganeyama Shrine for the use of their facilities, and Ms. Y. Sugiura for data collection. We also thank Prof. L. Fusani and an anonymous reviewer for their constructive comments. This work was supported by a Grant-in-Aid for Scientific Research (\#22570219) from JSPS to SH. 
464

465

466

467

468

\section{Figure Legends}

Figure 1. Initial numbers of individuals within $20 \mathrm{~m}$ from the focal individual ( $x$ axis) and changes in the number of individuals after 10 min ( $y$ axis), in the autumn (A), the winter (B), non-subgrouping in the summer (C), and subgrouping in the summer (D). A positive value indicates that the number of individuals increased, a negative value indicates that it decreased, and a value of zero indicates that it remained the same. A line represents the linear estimate by a generalised linear model, where the initial distance is the mean. Size of marks vary with the sample size (see legends in the figure), because multiple samples are plotted at the same point.

Figure 2. Initial distance between two individuals ( $x$ axis) and changes in distance apart after 10 min ( $y$ axis) in the autumn (A), the winter (B), non-subgrouping in the summer (C), and subgrouping in the summer (D). A positive value indicates that the distance apart became shorter, a negative value indicates that it became greater, and a value of zero indicates that it remained the same. A line represents the linear estimate by a generalised linear model, where the initial number is the mean.

Figure 3. Initial distance between two individuals ( $x$ axis) and mean coo-call rates during the following 1 min ( $y$ axis) in the autumn (A), the winter (B), non-subgrouping in the summer (C), and subgrouping in the summer (D). Error bars show 95\% confidence intervals of means.

Classes connected with bold lines showed a significant difference in post hoc pairwise comparisons ( $P<0.05$, panel B), and those connected with dashed lines showed a near-significant difference $(P=0.06$, panel A). 
488

489

490

491

492

493

494

495

496

497

498

499

500

501

502

503

504

505

506

507

508

509

510

511

512

\section{Literature Cited}

Agetsuma, N. 1995: Foraging synchrony in a group of Yakushima macaques (Macaca fuscata yakui). Folia Primatologica 64, 167-179.

Aoki, I. 1982: A simulation study on the schooling mechanism in fish. Bulletin of The Japanese Society of Scientific Fisheries 48, 1081-1088.

Aureli, F., Schaffner, C. M., Boesch, C., Bearder, S. K., Call, J., Chapman, C. A., Connor, R., Di Fiore, A., Dunbar, R. I. M., Henzi, S. P., Holekamp, K., Korstjens, A. H., Layton, R., Lee, P., Lehmann, J., Manson, J. H., Ramos-Fernandez, G., Strier, K. B. \& Van Schaik, C. P. 2008: Fission-fusion dynamics: New research frameworks. Current Anthropology 49, 627-654.

Boinski, S. \& Campbell, A. F. 1995: Use of trill vocalizations to coordinate troop movement among white-faced capuchins: A second field test. Behaviour 132, 875-901.

Cavagna, A., Cimarelli, A., Giardina, I., Parisi, G., Santagati, R., Stefanini, F. \& Tavarone, R. 2010: From empirical data to inter-individual interactions: Unveiling the rules of collective animal behavior. Mathematical Models \& Methods in Applied Sciences 20, 1491-1510.

D'Eon, R. G. \& Delparte, D. 2005: Effects of radio-collar position and orientation on GPS radio-collar performance, and the implications of PDOP in data screening. Journal of Applied Ecology 42, 383-388.

Green, S. 1975: Variation of vocal pattern with social situation in the Japanese monkey (Macaca fuscata): A field study. In: Primate Behavior, Vol. 4 (Rosenblum, L. A. ed). Academic Press, New York. pp. 1-102.

Hamilton, W. D. 1971: Geometry for selfish herd. Journal of Theoretical Biology 31, 295-311.

Hayakawa, S. 2007: Female defensibility in small troops of Japanese macaques vis-a-vis nontroop males and copulation on the periphery of the troop. International Journal Of Primatology 28, 73-96. 
Hill, D. A. \& Okayasu, N. 1995: Absence of "youngest ascendancy" in the dominance relations of sisters in wild Japanese macaques (Macaca fuscata yakui). Behaviour 132, 367-379.

Itani, J. 1963: Vocal communication of the wild Japanese monkey. Primates 4, 11-66.

Janson, C. H. 1990: Social correlates of individual spatial choice in foraging groups of brown capuchin monkeys, Cebus apella. Animal Behaviour 40, 910-921.

Karczmarski, L., Wursig, B., Gailey, G., Larson, K. W. \& Vanderlip, C. 2005: Spinner dolphins in a remote Hawaiian atoll: social grouping and population structure. Behavioral Ecology 16, 675-685.

Kazahari, N. \& Agetsuma, N. 2010: Mechanisms determining relationships between feeding group size and foraging success in food patch use by Japanese macaques (Macaca fuscata). Behaviour 147, $1481-1500$.

Koda, H., Shimooka, Y. \& Sugiura, H. 2008: Effects of caller activity and habitat visibility on contact call rate of wild Japanese macaques (Macaca fuscata). American Journal Of Primatology 70, 1055-1063.

Koda, H. \& Sugiura, H. 2010: The ecological design of the affiliative vocal communication style in wild Japanese macaques: Behavioral adjustments to social contexts and environments. In: The Japanese Macaques (Nakagawa, N., Nakamichi, M. \& Sugiura, H. eds). Springer, Tokyo. pp. 167-189.

Lukeman, R., Li, Y. X. \& Edelstein-Keshet, L. 2010: Inferring individual rules from collective behavior. Proceedings Of The National Academy Of Sciences Of The United States Of America 107, $12576-12580$.

Maruhashi, T., Saito, C. \& Agetsuma, N. 1998: Home range structure and inter-group competition for land of Japanese macaques in evergreen and deciduous forests. Primates 39, 291-301.

Mitani, M. 1986: Voiceprint identification and its application to sociological studies of wild Japanese monkeys (Macaca fuscata yakui). Primates 27, 397-412.

Ramos-Fernandez, G., Boyer, D. \& Gomez, V. P. 2006: A complex social structure with fission-fusion properties can emerge from a simple foraging model. Behavioral Ecology And Sociobiology 60, 536-549. 
Rendall, D., Cheney, D. L. \& Seyfarth, R. M. 2000: Proximate factors mediating "contact" calls in adult female baboons (Papio cynocephalus ursinus) and their infants. Journal of Comparative Psychology 114, 36-46.

Roberts, W. A. 2002: Are animals stuck in time? Psychological Bulletin 128, 473-489.

Robinson, J. G. 1981: Spatial structure in foraging groups of wedge-capped capuchin monkeys Cebus nigrivittatus. Animal Behaviour 29, 1036-1056.

Sato, S. 1988: Monkeys of the Kinkazan A group (in Japanese). Japanese Monkeys in Miyagi Prefecture 3, 6-29.

Schellinck, J. \& White, T. 2011: A review of attraction and repulsion models of aggregation: Methods, findings and a discussion of model validation. Ecological Modelling 222, 1897-1911.

Shimooka, Y. 2003: Seasonal variation in association patterns of wild spider monkeys (Ateles belzebuth belzebuth) at La Macarena, Colombia. Primates 44, 83-90.

Sugiura, H. 2007a: Adjustment of temporal call usage during vocal exchange of coo calls in Japanese macaques. Ethology 113, 528-533.

Sugiura, H. 2007b: Effects of proximity and behavioral context on acoustic variation in the coo calls of Japanese macaques. American Journal of Primatology 69, 1412-1424.

Sugiura, H., Shimooka, Y. \& Tsuji, Y. 2011: Variation in spatial cohesiveness in a group of Japanese macaques (Macaca fuscata). International Journal of Primatology 32, 1348-1366.

Suzuki, M. 2011: Monitoring behaviors for spatial cohesiveness of a group in wild Japanese macaques, Kyoto University.

Suzuki, M. \& Sugiura, H. 2011: Effects of Proximity and Activity on Visual and Auditory Monitoring in Wild Japanese Macaques. American Journal of Primatology 73, 623-631.

Tsuji, Y., Fujita, S., Sugiura, H., Saito, C. \& Takatsuki, S. 2006: Long-term variation in fruiting and the food habits of wild Japanese macaques on Kinkazan island, northern Japan. American Journal of Primatology 68, 1068-1080.

Tsuji, Y. \& Takatsuki, S. 2004: Food habits and home range use of Japanese macaques on an island inhabited by deer. Ecological Research 19, 381-388. 
Tsuji, Y. \& Takatsuki, S. 2012: Inter-annual variation in nut abundance is related to agonistic interactions of foraging female Japanese macaques (Macaca fuscata). Internatinal Journal of Primatology 33, 489-512.

van Schaik, C. P. \& van Noordwijk, M. A. 1986: The hidden costs of sociality: Intra-group variation in feeding strategies in Sumatran long-tailed macaques (Macaca fascicularis). Behaviour 99, 296-315.

Wada, K. \& Matsuzawa, T. 1986: A new approach to evaluating troop deployment in wild Japanese monkeys. International Journal of Primatology 7, 1.

Wrangham, R. W. 1980: An ecological model of female-bonded primate groups. Behaviour 75, 262-300.

Yamagiwa, J. \& Hill, D. A. 1998: Intraspecific variation in the social organization of Japanese macaques: Past and present scope of field studies in natural habitats. Primates 39, 257-273. 


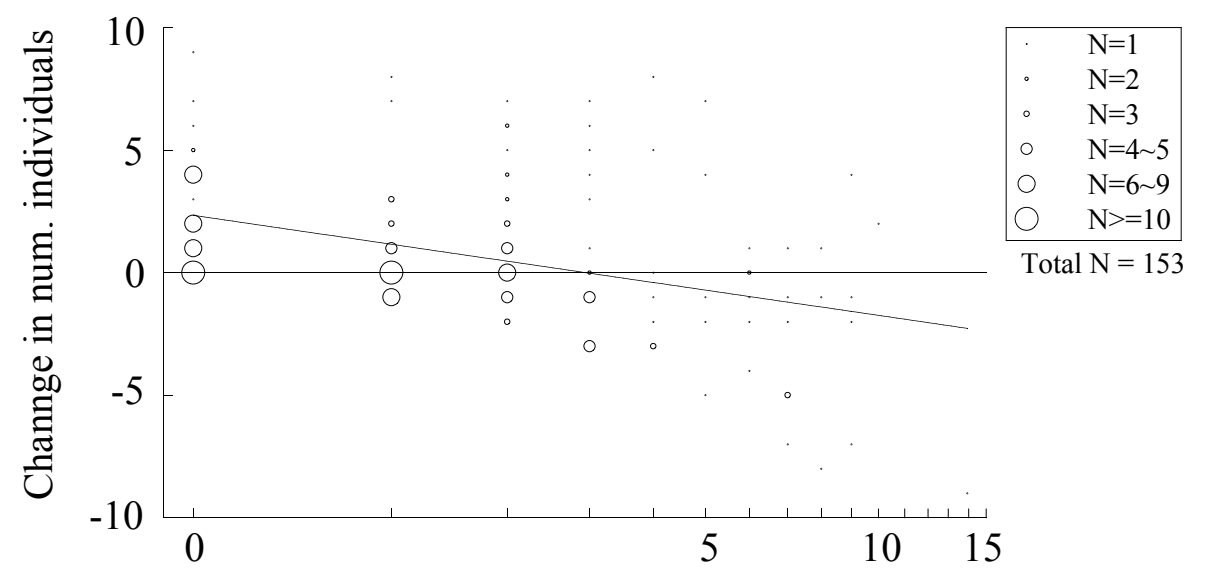

B) Winter

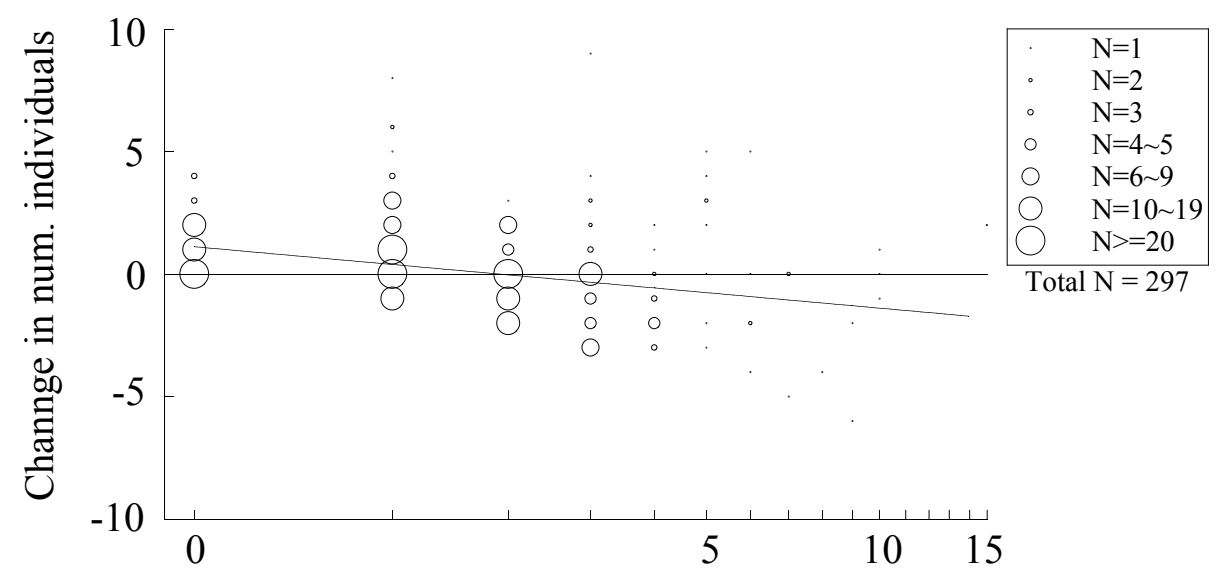

C) Summer, non-subgroup

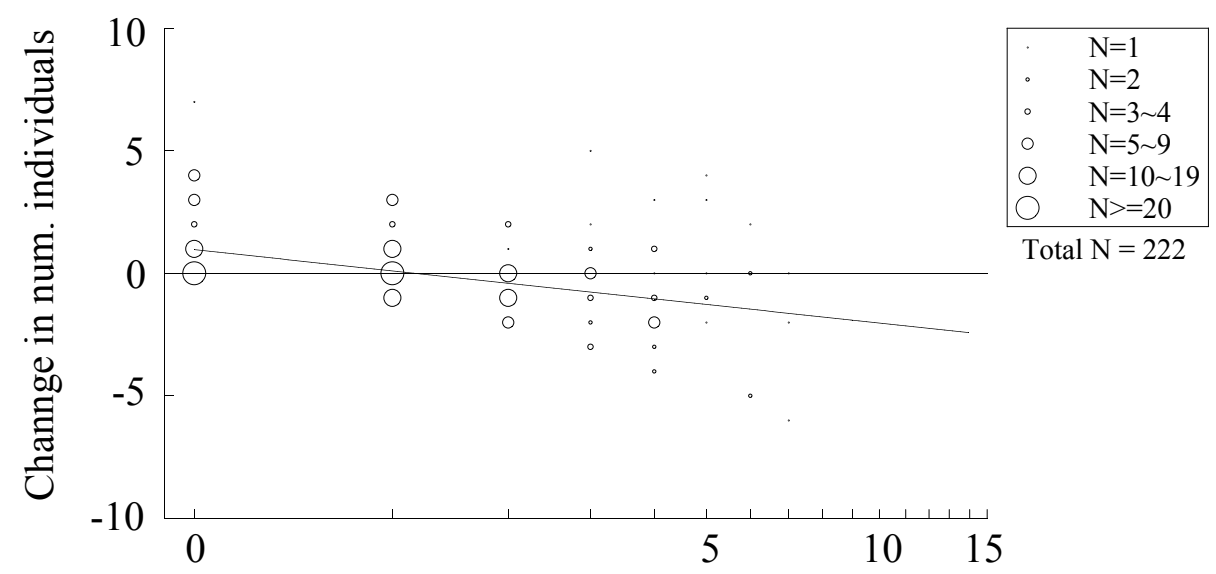

D) Summer, subgrouping

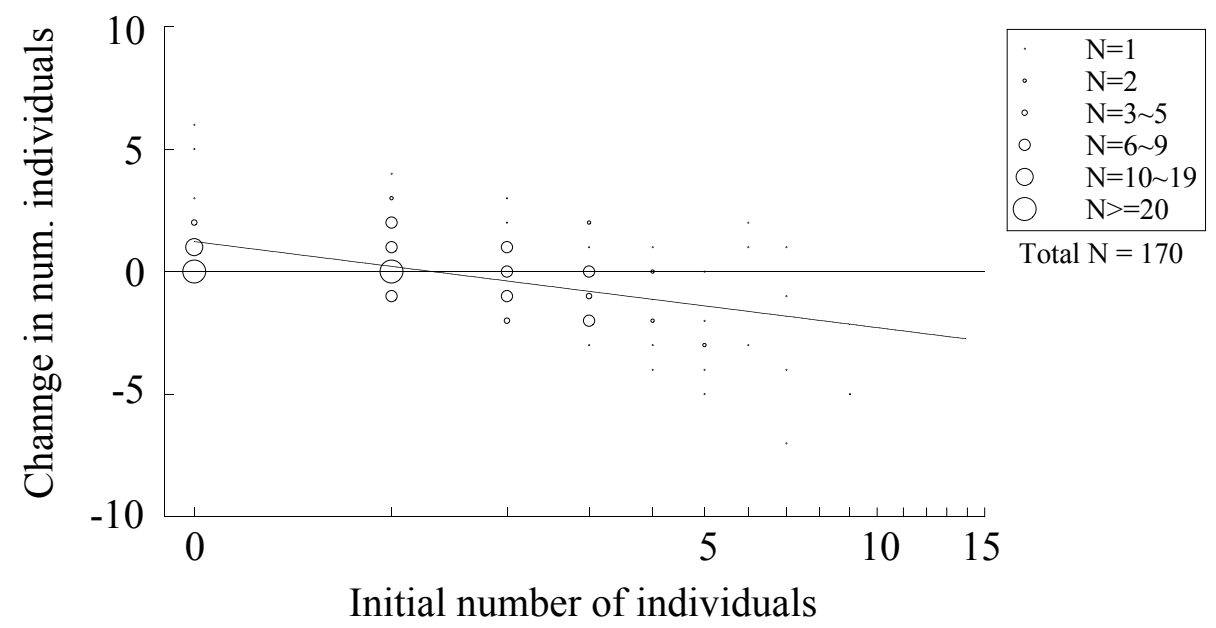

Fig. 1 


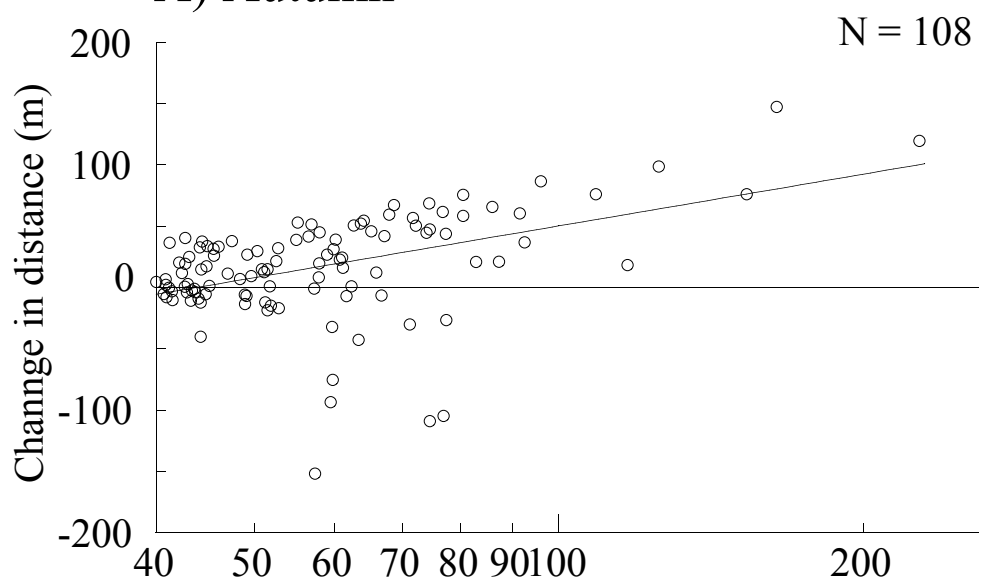

$$
\mathrm{N}=108
$$

B) Winter
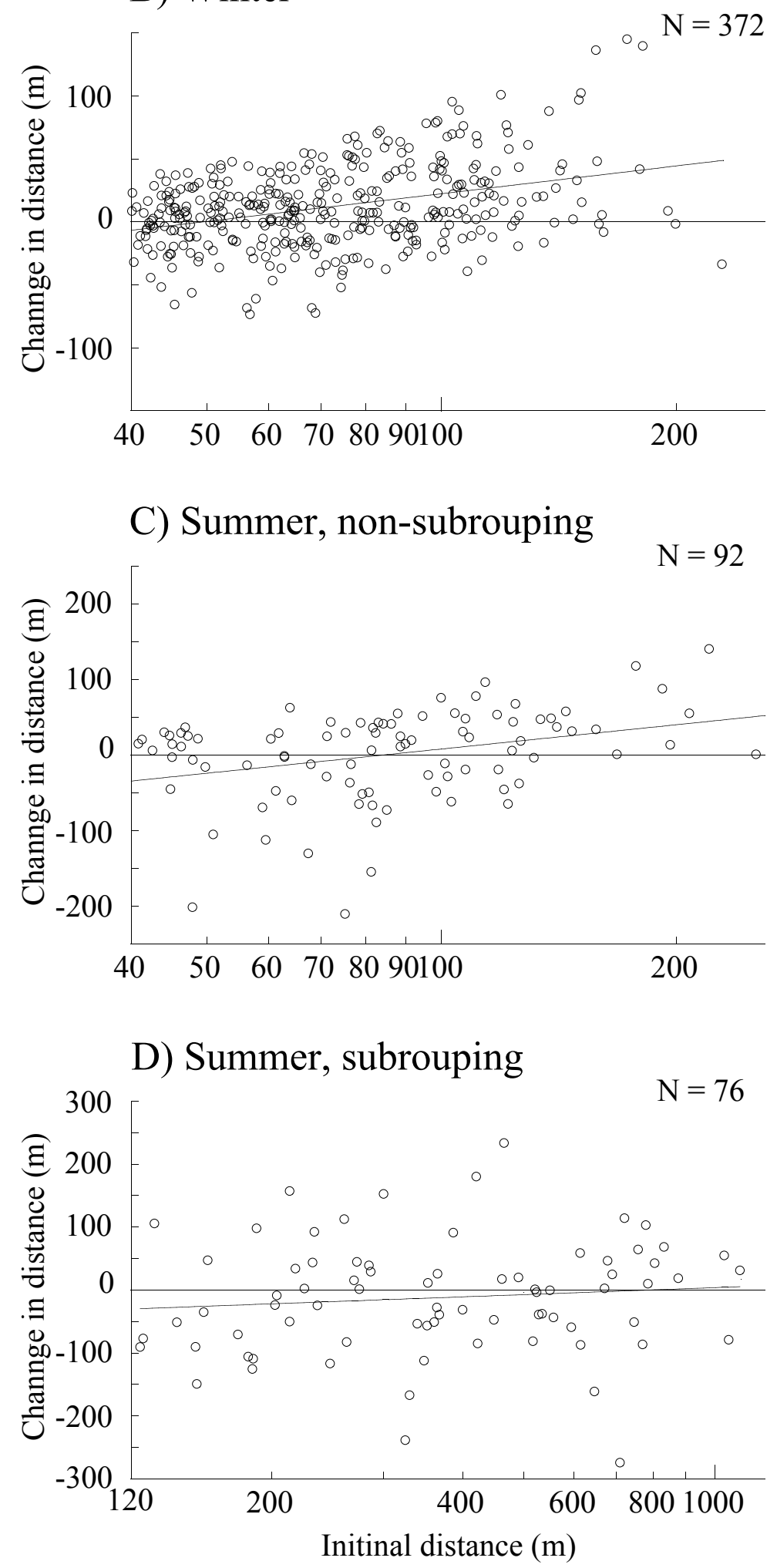

Fig. 2 
A) Autumn

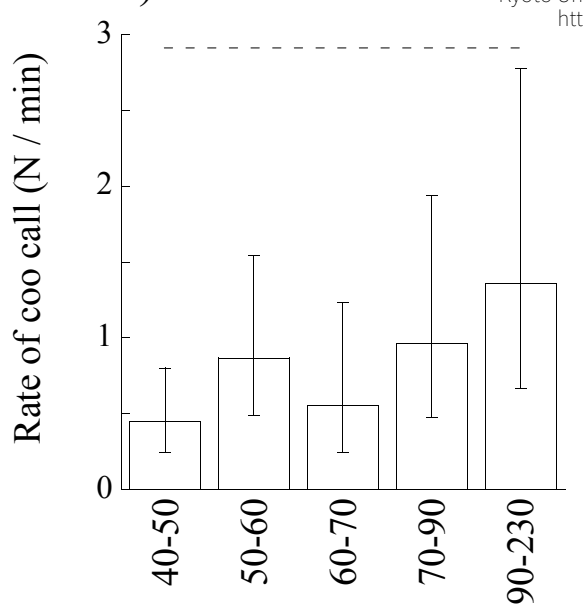

B) Winter

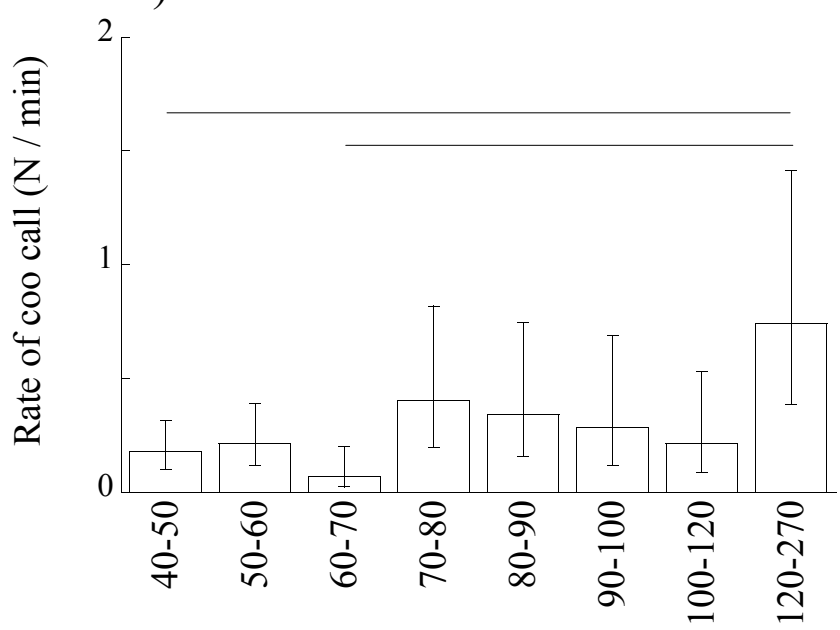

C) Summer, non-subgrouping

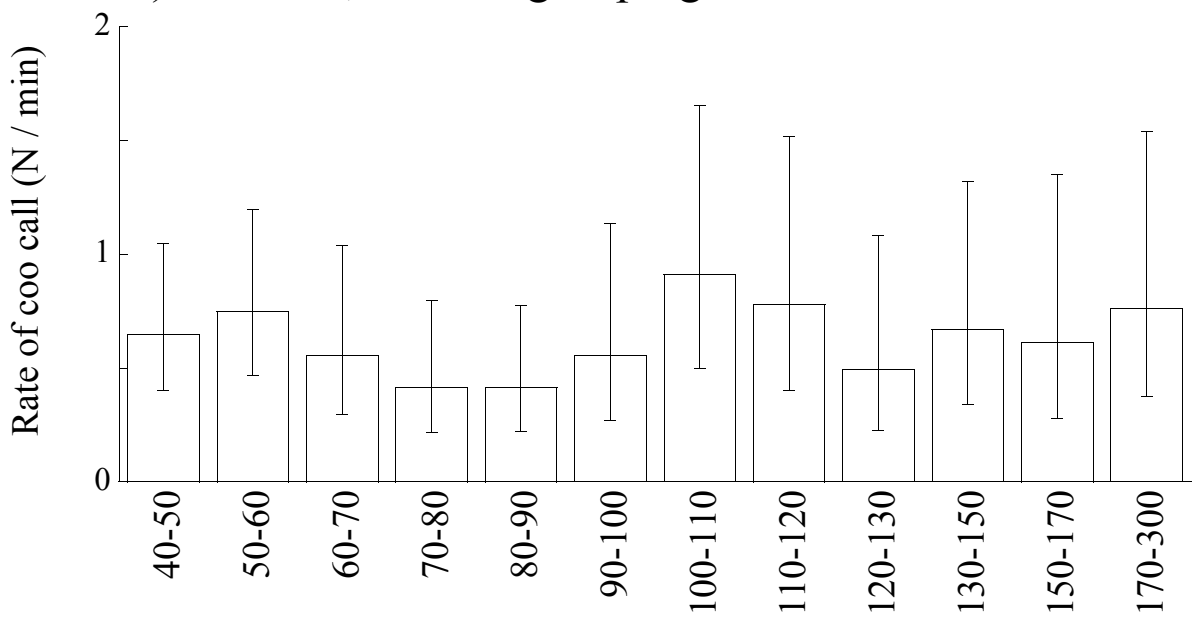

D) Summer, subgrouping

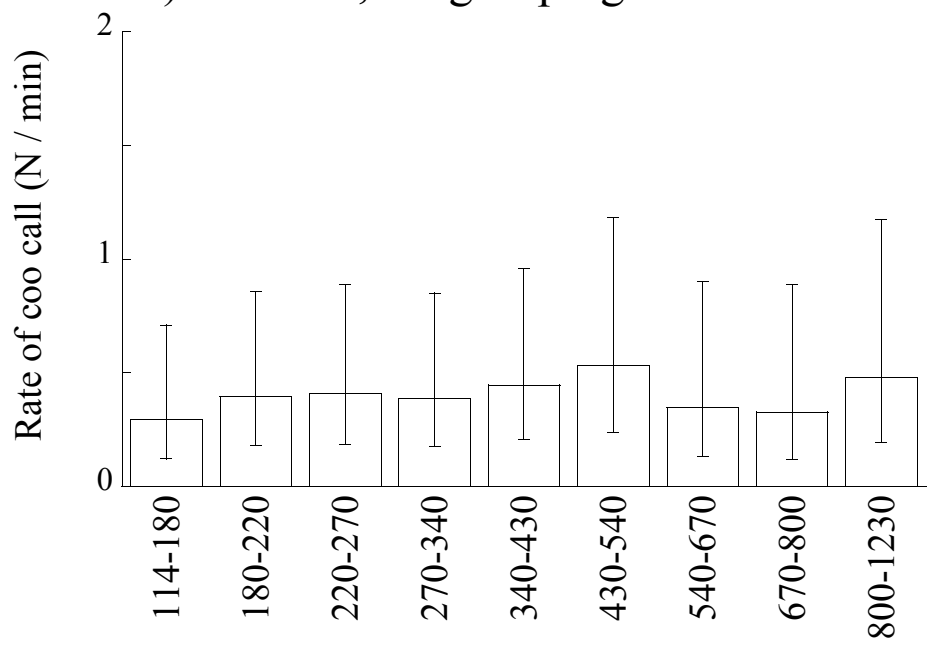

Fig. 3 